\title{
BMJ Open Coffee, including caffeinated and decaffeinated coffee, and the risk of hepatocellular carcinoma: a systematic review and dose-response meta-analysis
}

\author{
Oliver John Kennedy, ${ }^{1}$ Paul Roderick, ${ }^{1}$ Ryan Buchanan, ${ }^{1}$ \\ Jonathan Andrew Fallowfield, ${ }^{2}$ Peter Clive Hayes, ${ }^{2}$ Julie Parkes ${ }^{1}$
}

To cite: Kennedy 0J, Roderick $P$, Buchanan $\mathrm{R}$, et al. Coffee, including caffeinated and decaffeinated coffee, and the risk of hepatocellular carcinoma: a systematic review and dose-response meta-analysis. BMJ Open 2017;7:e013739. doi:10.1136/ bmjopen-2016-013739

- Prepublication history and additional material are available. To view please visit the journal (http://dx.doi.org/ 10.1136/ bmjopen-2016-013739).

Received 3 August 2016 Revised 30 January 2017 Accepted 6 March 2017

CrossMark

${ }^{1}$ Primary Care and Population Sciences Faculty of Medicine, University of Southampton, Southampton, UK

${ }^{2}$ MRC Centre for Inflammation Research, University of Edinburgh, Edinburgh, UK

Correspondence to Dr Oliver John Kennedy; ok4g13@soton.ac.uk

\section{ABSTRACT}

Objectives To examine the association between coffee, including caffeinated and decaffeinated coffee, with hepatocellular carcinoma (HCC) and assess the influence of HCC aetiology and pre-existing liver disease.

Design We performed a systematic review and metaanalysis. We calculated relative risks (RRs) of HCC according to caffeinated and decaffeinated coffee consumption using a random-effects dose-response meta-analysis. We tested for modification of the effect estimate by $\mathrm{HCC}$ aetiology and pre-existing liver disease. We judged the quality of evidence using the Grading of Recommendations Assessment, Development and Evaluation (GRADE) criteria.

Results We found 18 cohorts, involving 2272642 participants and 2905 cases, and 8 case-control studies, involving 1825 cases and 4652 controls. An extra two cups per day of coffee was associated with a $35 \%$ reduction in the risk of $\mathrm{HCC}$ ( $\mathrm{RR} 0.65,95 \% \mathrm{Cl} 0.59$ to 0.72 ). The inverse association was weaker for cohorts (RR $0.71,95 \% \mathrm{Cl}$ 0.65 to 0.77 ), which were generally of higher quality than case-control studies (RR $0.53,95 \% \mathrm{Cl} 0.41$ to 0.69 ). There was evidence that the association was not significantly altered by stage of liver disease or the presence/absence of high alcohol consumption, high body mass index, type 2 diabetes mellitus, smoking, or hepatitis $B$ and $C$ viruses. An extra two cups of caffeinated and decaffeinated coffee ( 2 and 3 cohort studies, respectively) were associated with reductions of $27 \%$ (RR $0.73,95 \% \mathrm{Cl} 0.63$ to 0.85 ) and $14 \%$ (RR $0.86,95 \% \mathrm{Cl} 0.74$ to 1.00 ) in the risk of HCC. However, due to a lack of randomised controlled trials, potential publication bias and there being no accepted definition of coffee, the quality of evidence under the GRADE criteria was 'very low'.

Conclusions Increased consumption of caffeinated coffee and, to a lesser extent, decaffeinated coffee are associated with reduced risk of HCC, including in pre-existing liver disease. These findings are important given the increasing incidence of HCC globally and its poor prognosis.

\section{INTRODUCTION}

Primarylivercancer is the sixth mostcommonly diagnosed cancer worldwide, and because of its poor prognosis the second leading cause of cancer death. ${ }^{12}$ Hepatocellular carcinoma

\section{Strengths and limitations of this study}

This is the first meta-analysis to calculate the relative risks (RRs) of hepatocellular carcinoma (HCC) for 1-5 cups of coffee per day, which may be useful in the design of a coffee-based intervention for evaluation in a clinical trial.

- This is the first meta-analysis to investigate the influence of all the main HCC risk factors on the association between coffee and HCC.

- This is the first meta-analysis to calculate the RR of HCC for decaffeinated coffee consumption.

- There was heterogeneity between the studies included in the meta-analysis.

Many studies did not specify coffee caffeine content.

(HCC) is the dominant histological subtype accounting for $85 \%-90 \%$ of cases. ${ }^{3}$ HCC most commonly develops in people with cirrhosis due to chronic viral hepatitis $\mathrm{B}$ (HBV) or hepatitis C (HCV), excess alcohol consumption, and/or non-alcoholic fatty liver disease. ${ }^{3}$ Non-alcoholic steatohepatitis (NASH), which is rapidly increasing worldwide, can lead to the development of HCC in the absence of cirrhosis. ${ }^{4}$ The incidence of liver cancer is increasing due to changes in these underlying risks, and by 2030 the number of new cases annually will have risen by around $50 \%$ to over 1.2 million. ${ }^{5}$ The burden of liver cancer is highest in East and South-East Asia, with China alone accounting for $50 \%$ of cases worldwide. $^{2}$ Only $10 \%-37 \%$ of patients diagnosed with HCC are eligible for potentially curative tumour resection (partial hepatectomy).$^{6}$ Thus, prognosis remains poor, with a 5 -year overall survival rate of $18 \%$.

Coffee is a popular drink in most countries, with approximately 2.25 billion cups consumed daily. ${ }^{8}$ It is a complex mixture of biologically active molecules, including caffeine, chlorogenic acid and diterpenes. 
These compounds possess antioxidant, anti-inflammatory, antifibrotic and anticarcinogenic properties, which may explain the observational data that coffee drinkers have lower rates of chronic liver disease (CLD), including fibrosis, cirrhosis and HCG. ${ }^{10}$ Reports by the World Cancer Research Fund (WCRF) ${ }^{11}$ and the International Agency for Research on Cancer (IARC) ${ }^{12}$ are both supportive of a protective role of coffee against HCC. In addition, a recent meta-analysis reported that the relative risk (RR) of HCC for an extra cup of coffee per day was $0.74(95 \%$ CI 0.65 to 0.83$) .{ }^{13}$ However, to date no randomised controlled trials (RCTs) investigating a coffee intervention for preventing HCC have been performed. Challenges in designing such a trial include a lack of understanding of the effect modification by aetiology or risk factors for HCC (eg, alcohol liver disease, NASH, cirrhosis, etc). In addition, there is uncertainty as to whether all types of coffee are equally beneficial, especially given their differing chemical compositions (eg, caffeinated vs decaffeinated coffee). To help address these challenges, we have now explored, for the first time in a meta-analysis, the modification of the inverse association between coffee and HCC by key risk factors, such as $\mathrm{HBV} / \mathrm{HCV}$, high body mass index (BMI), type 2 diabetes mellitus (T2DM), smoking, alcohol consumption and the presence of CLD including cirrhosis. We also report the first meta-analysis for the association between decaffeinated coffee and HCC. Decaffeinated coffee protects against liver damage in animal studies ${ }^{14}$ and is inversely associated with T2DM, abnormal liver function tests and cirrhosis in human observational studies. ${ }^{15-17}$

\section{METHODS}

The methods used were similar to those described in our earlier work ${ }^{18}$ and are detailed below. We followed the PRISMA (Preferred Reporting Items for Systematic Reviews and Meta-Analyses) guidelines; a protocol, which was prespecified but not preregistered online, is provided as online supplementary file information.

\section{Searches and selection of studies}

We performed searches of abstracts and titles in Web of Science, Embase and PubMed with the following: ('odds' OR 'risk' OR 'hazard' OR 'OR' OR 'RR' OR 'HR') AND 'coffee' AND ('liver' OR 'hepatocellular*') AND ('cancer 'OR 'carcino*' OR 'neoplas*'). The searches were run in September 2015 without restriction of date of publication. References of pertinent studies were searched manually. After removing duplicates, OK and RB independently screened the titles and abstracts of the studies found in the search. Studies that were included (1) reported an RCT, case-control study or cohort study; and (2) reported HRs, ORs or RRs with 95\% CIs for HCC in adults according to consumption of coffee. Studies that were excluded (1) did not report a dose-response or give sufficient information for calculation of a dose-response (ie, this requires estimates for more than two exposure levels, or (ii) were non-English-language studies. We assumed cases of primary liver cancer to be HCC. If studies overlapped, we included the largest study or otherwise the last published study. We worked from published studies only, including abstracts, although we unsuccessfully attempted to acquire unpublished data from the authors of one study, as indicated below.

\section{Extraction of data and assessment of quality}

We extracted the following information from each study: the first author, the date of publication, the geographic region, the design of the study, the exclusion and inclusion criteria, the estimates and adjustments, the numbers of participants (or controls) and cases, the methods of measuring exposure, and case identification. We also extracted data concerning cohort follow-up (time, losses) and whether baseline liver disease was excluded. We extracted the most rigorously adjusted effect sizes. We extracted effect sizes stratified by pre-existing CLD, smoking status, alcohol consumption, BMI, HBV and HCV status, T2DM, and type of coffee. OK extracted the data, which RB then checked. Given the low incidence of HCC, we considered ORs, RRs and HRs to be equivalent, and for simplicity we used RR to refer to all three herein. We assessed the quality of the included studies using the Newcastle-Ottawa Scale. ${ }^{19}$ We judged the quality of evidence with the Grading of Recommendations Assessment, Development and Evaluation (GRADE) ${ }^{20}$

\section{Statistical methods \\ Coffee and HCC}

Most studies did not distinguish caffeinated versus decaffeinated coffee, so coffee was taken to be the pattern of use prevalent in the particular study population. We considered consumption in cups, where necessary ${ }^{21}$ converting millilitres into cups of $150 \mathrm{~mL}$. For each study, we calculated an RR for an extra two cups per day using dose-response data where available ${ }^{22} 23$ or by estimating the dose-response using the method of Greenland and Longnecker. ${ }^{24}$ The unit of an 'extra two cups' per day was selected to represent a potential coffee-based intervention, which could be used in clinical trials, and to maintain comparability with a previous meta-analysis. ${ }^{25}$ We estimated the median consumption for each reported consumption category to be the midpoint of closed ranges and the midpoint added to the amplitude of the previous range for open ranges. ${ }^{25} \mathrm{We}$ assessed whether the dose-response was non-linear by a cubic spline meta-analysis. ${ }^{26}$ We tested for statistical heterogeneity using $\mathrm{I}^{2}$ and Cochran's $Q^{27}$ and interpreted $p$ values of $<0.1$ as statistically significant (for heterogeneity only), and we interpreted the $\mathrm{I}^{2}$ values according to chapter 9.5.2 of the Cochrane handbook. ${ }^{27}$ We investigated heterogeneity by meta-regression and examined the impact of individual studies by rerunning the analysis while leaving the studies out one at a time..$^{28}$ We tested for publication bias using Egger's test and a 'trim-and-fill' analysis, ${ }^{29}$ which we used to adjust the estimate for missing studies if publication 
bias was indicated. To assess the magnitude and direction of adjustment, we calculated a pooled unadjusted effect sizes for comparison with the corresponding adjusted effect size. We used random-effects models (DerSimonian-Laird) and a two-sided $p$ value $>0.05$ for statistical significance. We used R (R Foundation for Statistical Computing, Vienna, Austria) with the metafor ${ }^{30}$ and dosresmeta ${ }^{31}$ packages for the analyses.

\section{Effect modification by risk factors}

We calculated the RRs of HCC according to coffee consumption in participants stratified by baseline CLD. We also calculated and meta-analysed RRs stratified by exposure to each of viral hepatitis status (carriers of $\mathrm{HBV} / \mathrm{HCV}$ vs negative for both), BMI (highest vs lowest BMI categories), T2DM (presence vs absence), alcohol consumption (highest vs lowest categories) and smoking (current smoker vs ex/non-smoker). For these analyses, we only included studies that provided RRs for both exposed and non-exposed to the risk factors. Where available, ${ }^{22} 23$ we used dose-response data to calculate RRs for an increase in two cups of coffee per day. Otherwise, we used the Greenland and Longnecker method, ${ }^{24}$ where the number of exposed and non-exposed was provided ${ }^{32-35}$ and variance-weighted least squares regression where they were not. ${ }^{36-39}$ For each risk factor, we calculated a $p$ value for its modifying effect on the association between coffee and HCC by meta-analysing the differences between the exposed and unexposed RRs from each study. We also calculated the $\tau^{2}$ for each of these analyses.

\section{Caffeinated and decaffeinated coffee and HCC}

Where possible we extracted data separately for caffeinated and decaffeinated coffee and calculated pooled RRs of HCC per two extra cups per day of each. One study, Bamia et $a l,{ }^{21}$ reported RRs of HCC according to decaffeinated coffee consumption for three qualitative categories: 'non-consumers', 'consumers below the median' and 'consumers at/above the median'. We were unable to get the corresponding quantitative values after contacting the authors, so we used those reported by another publication investigating the effect of decaffeinated coffee on oesophageal cancer in the same cohort. ${ }^{40}$ As above, we used dose-response data where available. ${ }^{22}$ Otherwise, we calculated the dose-response using the Greenland and Longnecker method, ${ }^{24}$ where the number of exposed and non-exposed ${ }^{15}$ was available and variance-weighted least squares regression where they were not. ${ }^{21}$

\section{RESULTS}

\section{Coffee consumption and HCC}

Figure 1 shows the searches and the stages of the selection of studies. Once duplicates were removed, we screened the abstracts and titles of 181 studies. Of those, we reviewed 34 studies in their entirety. Tables $1 \mathrm{~A}$ and $1 \mathrm{~B}$ summarise the characteristics of the 16 studies that we included in the main meta-analysis. 15 21-23 3233 35-39 41-45 The studies were published between 2002 and 2015.
Seven were from Europe, five from Japan, two from the USA and one from each of Hong Kong and Singapore. The cohort studies primarily involved general populations (eg, randomly selected from population registries), except for Lai $e t a l,{ }^{23}$ which included male smokers only. Total follow-ups ranged from $7^{39}$ to 24 years, ${ }^{23}$ and linkage to cancer registries was generally used to identify cases and exclude baseline HCC. The case-control studies were hospital-based, with only one ${ }^{33}$ using community controls. Fifteen studies reported estimates according to 'coffee' consumption, while two and four studies, respectively, reported estimates specifically for caffeinated and decaffeinated coffee. The quality scores ranged from 4 to 8 (tables 1A and 1B) and were generally higher for cohorts (mean=6.9) compared with case-control studies (mean=5.0). A number of studies reported data from multiple cohorts or case-control studies. We extracted pooled estimates from Petrick $e t a l^{22}$ (nine cohorts) and Gallus $e t a l^{37}$ (two case-control studies) as equivalent study-specific estimates (eg, in terms of adjustments for confounders and categories of coffee consumption) were not available. We extracted separate RRs from Shimazu $e t$ $a l^{39}$ (two cohorts). Thus, this meta-analysis included data from 18 cohorts, involving 2272642 participants and 2905 cases, and 8 case-control studies, involving 1825 cases and 4652 controls.

The RRs of HCC according to coffee consumption are summarised in table 2, including adjustments for confounders. Most studies adjusted for age, alcohol and smoking, and a smaller number for $\mathrm{HBV} / \mathrm{HCV}$, BMI and T2DM. All the studies showed an inverse association between HCC for an extra two cups of coffee per day, although in four studies the relationship was not statistically significant. The pooled RR of HCC for an extra two cups per day across all studies for coffee was 0.65 (95\% CI 0.59 to 0.72 ) (figure 2), for cohort studies it was 0.71 (95\% CI 0.65 to 0.77 ) and for case-control studies 0.53 (95\% CI 0.41 to 0.69 ). The pooled RR from studies with a quality score of 6 or above was 0.70 (95\% CI 0.64 to 0.76 ) compared with 0.50 (95\% CI 0.35 to 0.70 ) for those scoring below 6 . The $p$ value for non-linearity of the dose-response was not statistically significant, and the pooled RRs for different levels of consumption of up to five cups per day are illustrated in figure 3. Adjustment for confounders had minimal effect, changing the pooled RR from 0.62 (95\% CI 0.53 to 0.72 ) (ie, unadjusted) to 0.65 (95\% CI 0.59 to 0.72 ).

\section{Heterogeneity and sensitivity analysis}

The $\mathrm{I}^{2}$ and the $\mathrm{p}$ value for Cochran's $\mathrm{Q}$ were $58.5 \%$ and $<0.01$, respectively (figure 2), which indicated 'moderate' to 'substantial' between-study heterogeneity. Heterogeneity was lower for cohorts $\left(I^{2}=40.7 \% ; \mathrm{p}=0.09\right)$ than case-control studies $\left(\mathrm{I}^{2}=64.3 \%\right.$; $\left.\mathrm{p}<0.01\right)$. In the sensitivity analysis, the RR was strongest when we excluded $\mathrm{Hu} e t a l^{8}$ (RR $0.63,95 \% \mathrm{CI} 0.56$ to 0.71 ) and weakest when we excluded Tanaka $e t a l^{33}$ (RR $0.68,95 \%$ CI 0.62 to 0.74$)$. Heterogeneity remained statistically significant 


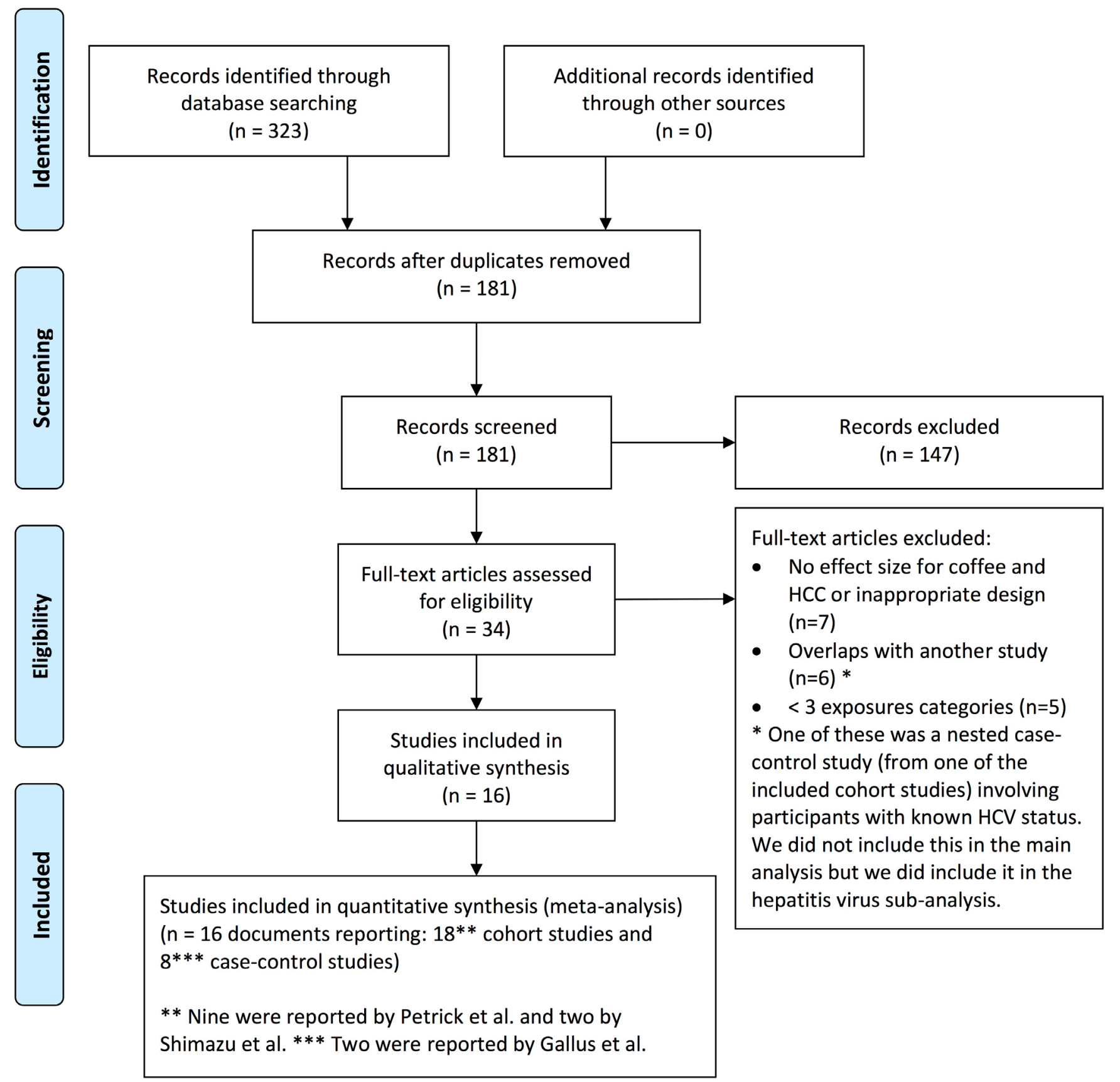

Figure 1 An illustration showing how the studies included in this meta-analysis were reviewed and selected. HCC, hepatocellular carcinoma; $\mathrm{HCV}$, hepatitis $\mathrm{C}$ virus.

throughout. In the meta-regression analysis, we found no statistically significant association of RR and publication year, length of follow-up (cohorts only), percentage of alcohol abstainers, age or gender.

\section{Publication bias and quality of evidence}

We found evidence of publication bias by Egger's test $(p<0.0001)$ and visual inspection of the funnel plot as shown in figure 4. In our trim-and-fill analysis, we detected a number of 'missing' smaller studies. Calibration for missing studies pushed the effect size of coffee towards null from 0.65 (95\% CI 0.59 to 0.72$)$ to 0.71 (95\% CI 0.64 to 0.79 ). The evidence quality that coffee protects against HCC as determined with GRADE was 'very low' (table 3).

The effect of pre-existing CLD and HCC risk factors

Three cohort studies ${ }^{3536} 39$ performed subgroup analyses stratified by presence/absence of baseline CLD, which was poorly defined but included cirrhosis. Data from two of those studies showed an inverse association of coffee and HCC in those with baseline CLD but not without, while the other showed an inverse association without baseline CLD only. The pooled difference between the stratified estimates was not statistically significant $(\mathrm{p}=0.87)$. 


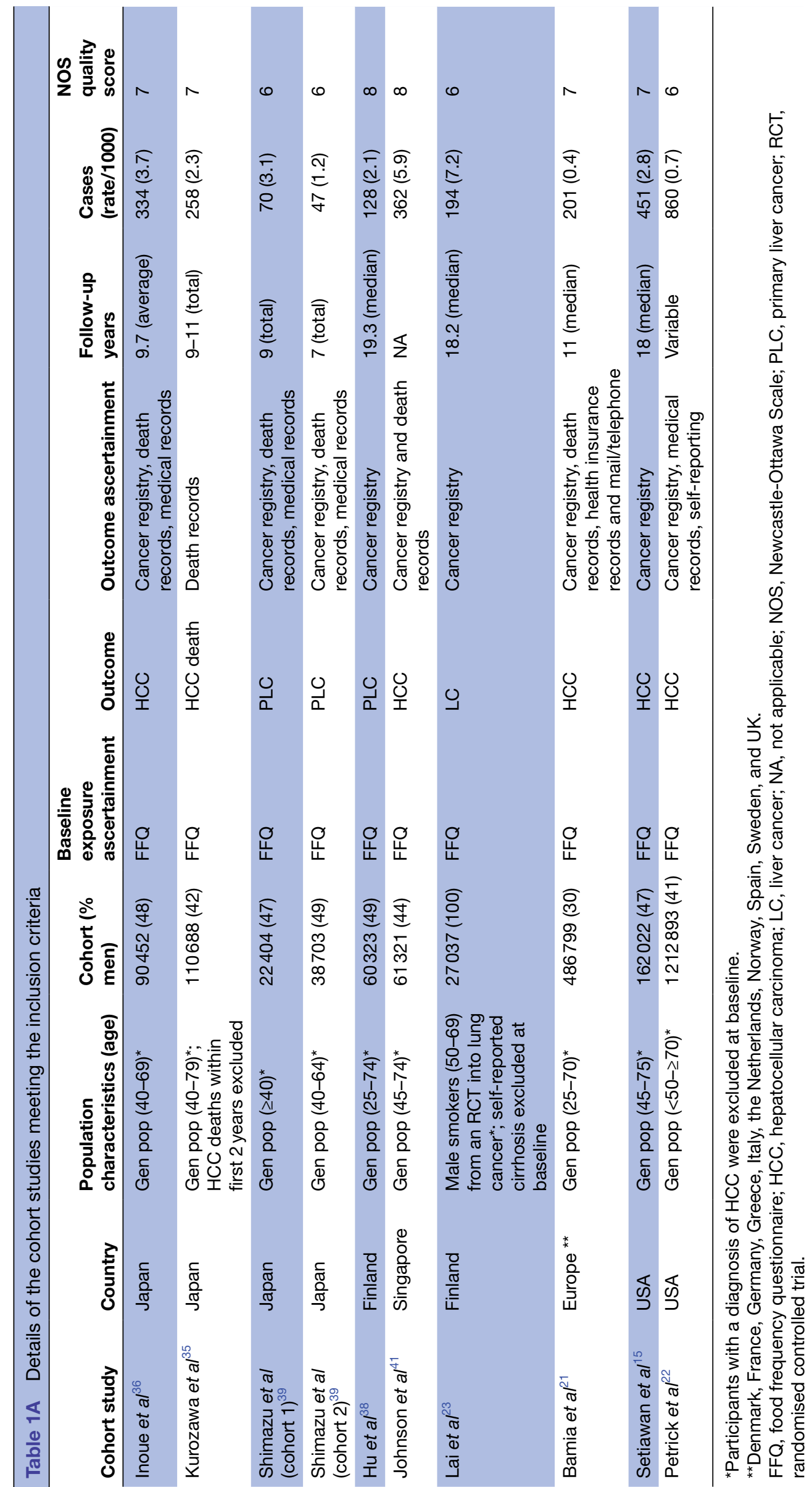




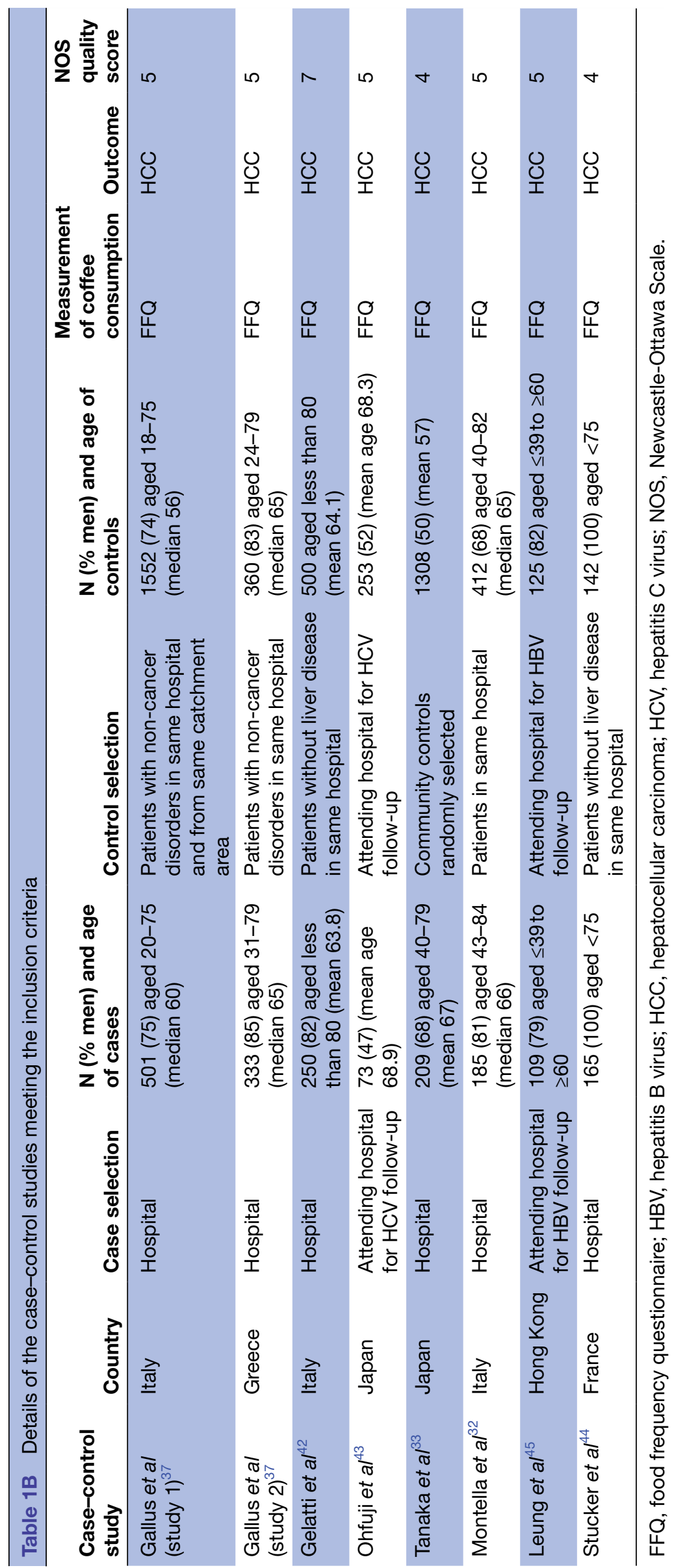



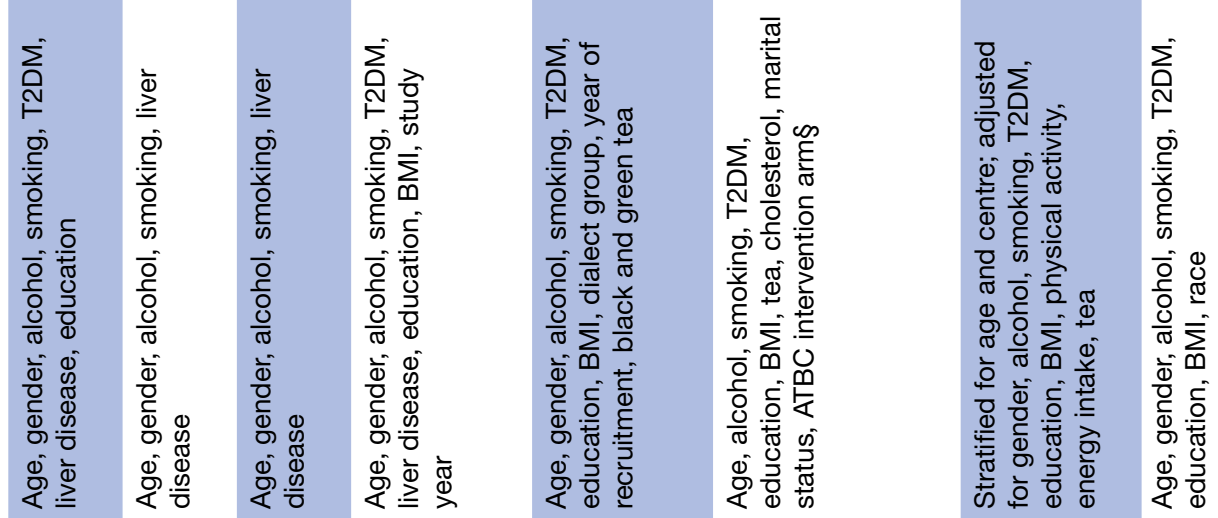

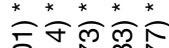

莡

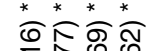

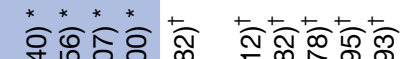

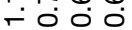

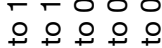

50
$\circ$

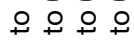

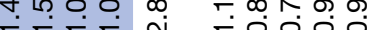

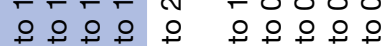

ลั.

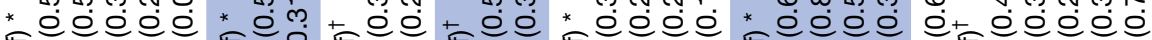

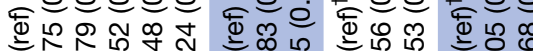

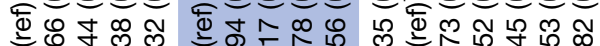
t. + + 뉴요 유유 은

$\infty$ 我

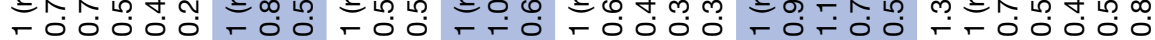

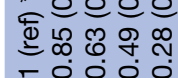

*으응 di

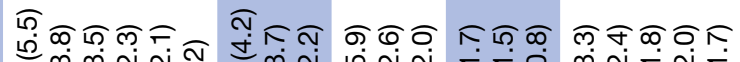

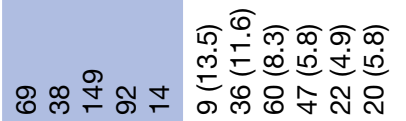

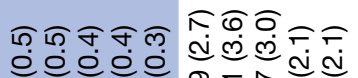
$\overline{0}$
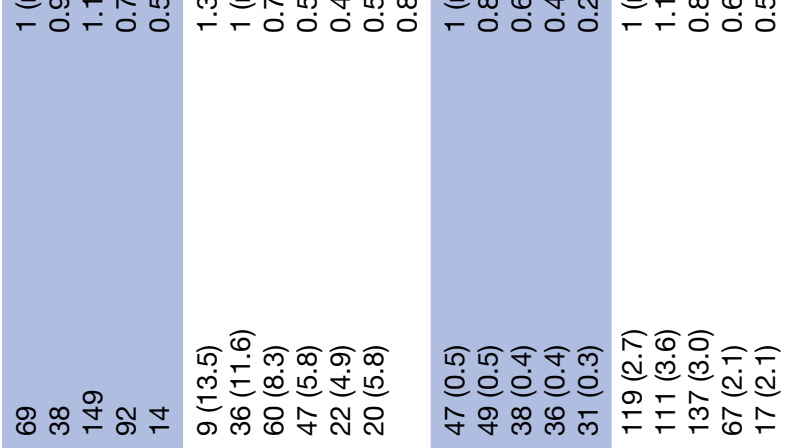

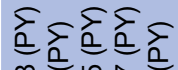

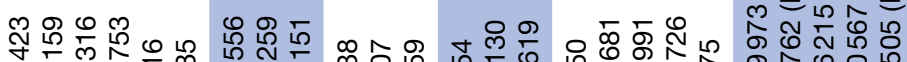

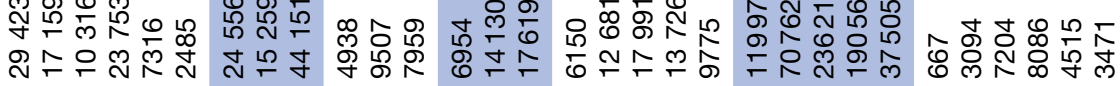

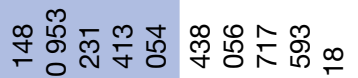

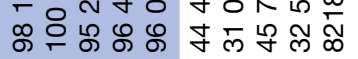

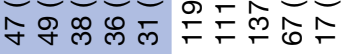
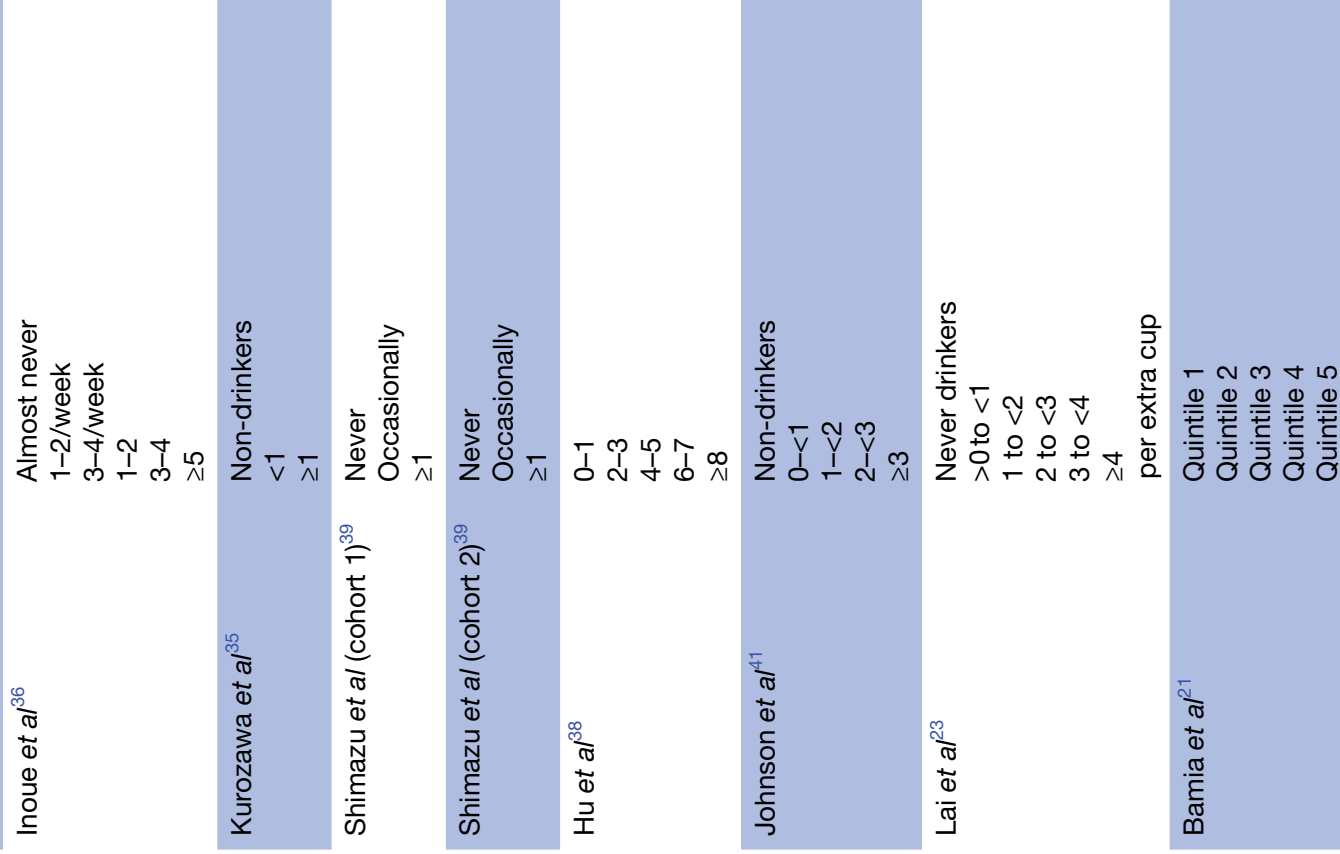

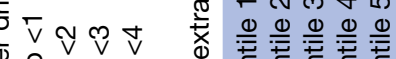

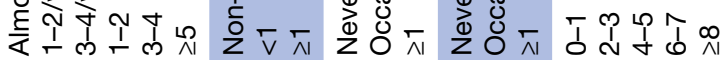
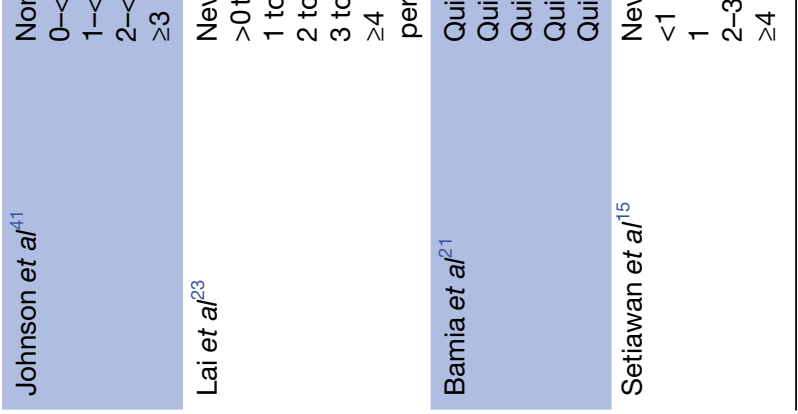


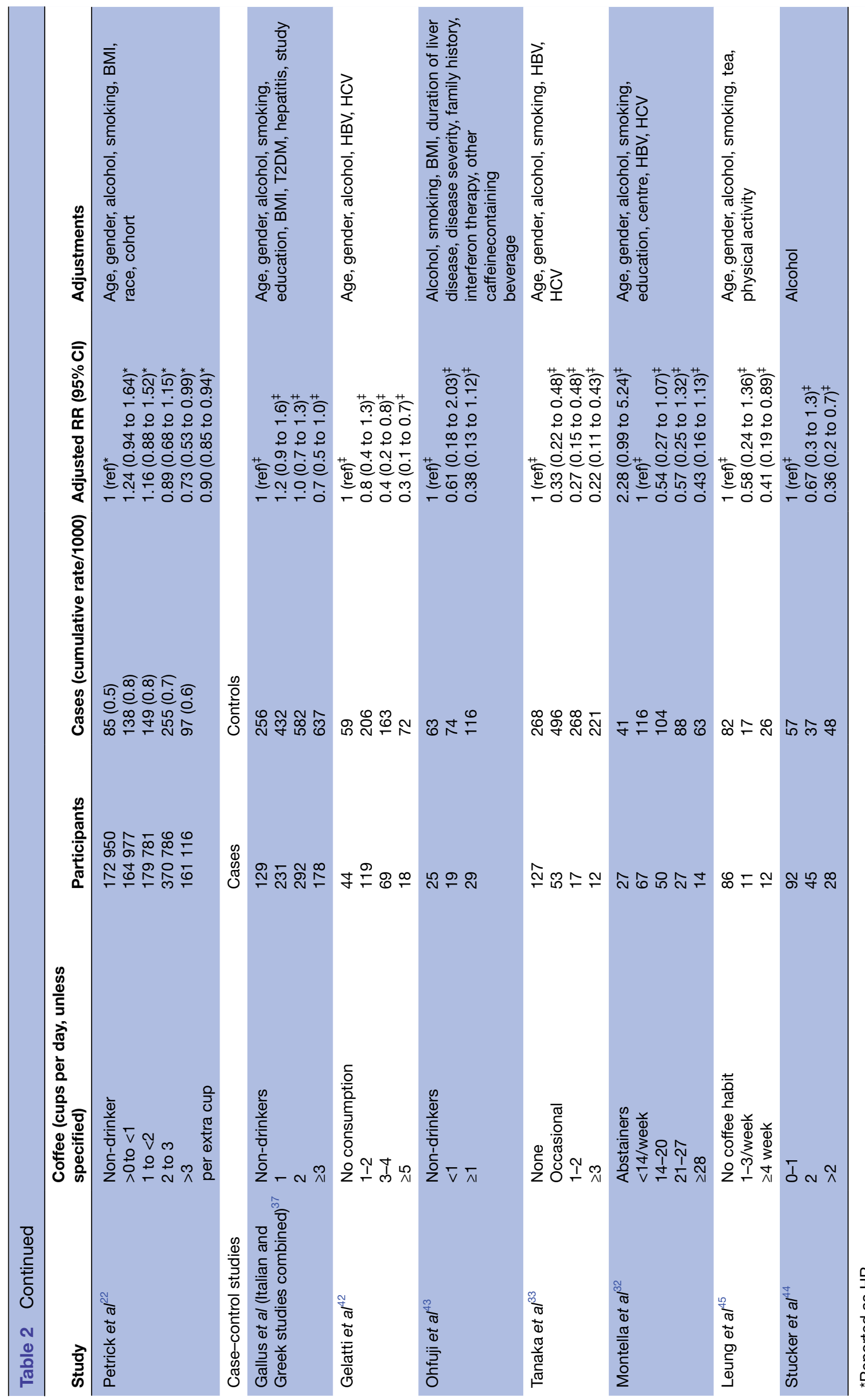




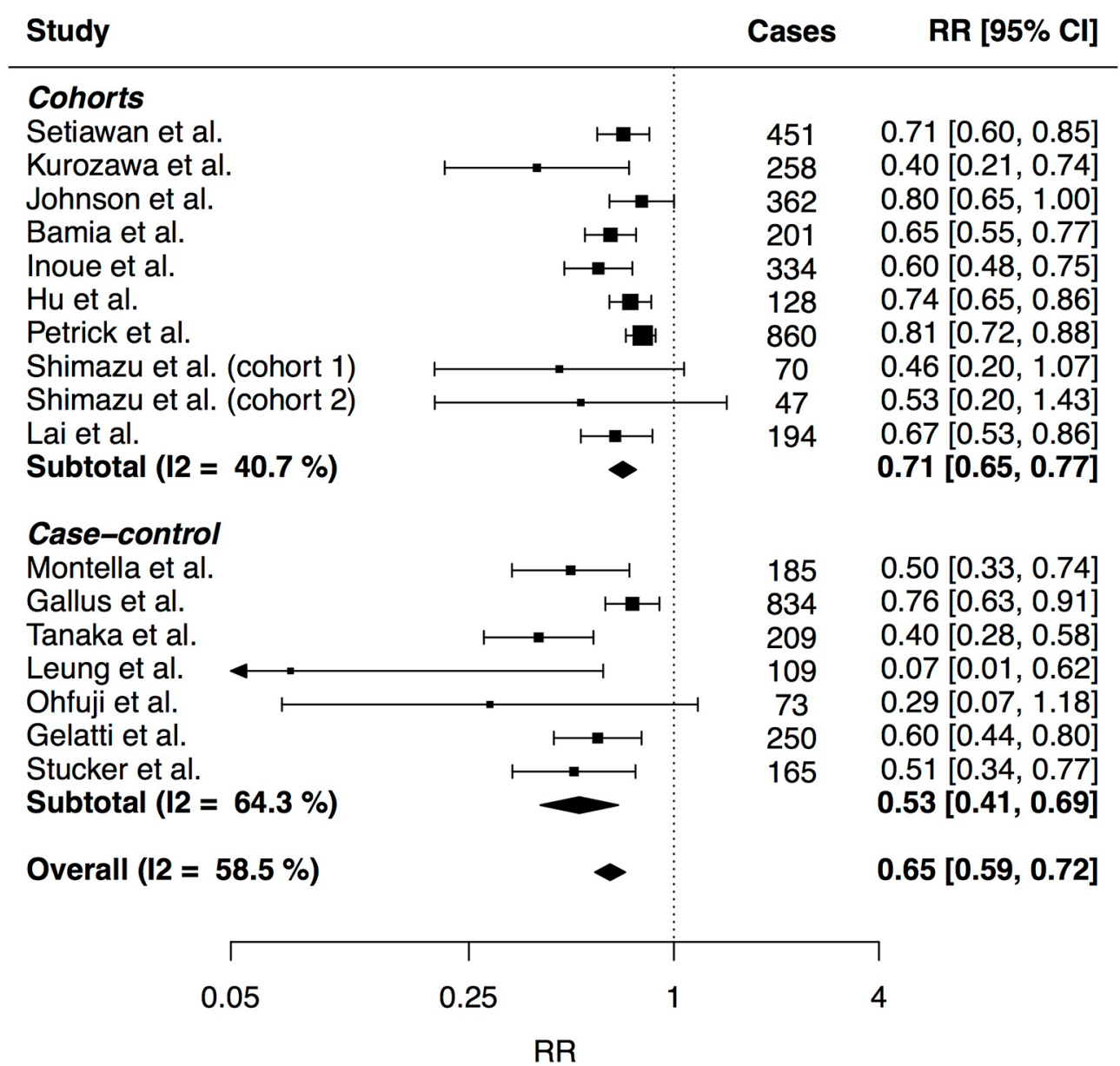

Figure 2 A forest plot illustrating RRs of HCC for an extra two cups of coffee per day. The RRs as reported by the individual studies are shown as squares. The sizes of the squares represent the weightings in the random-effects model. The pooled RRs (from cohorts, case-control studies and all studies) are shown as diamonds. HCC, hepatocellular carcinoma; RR, relative risks.

Data from a fourth (case-control) study ${ }^{33}$ showed statistically significant inverse associations between coffee and HCC, both when cases were compared with community controls and controls with CLD, $22 \%$ of whom had cirrhosis. Three other case-control studies ${ }^{374} 43$ showed inverse associations between coffee and HCC using only controls with liver disease.

Results from the investigation into the influence of risk factors on the association between coffee and HCC are presented as online supplementary file information. In summary, there was no statistically significant difference in the associations between coffee and HCC according to viral hepatitis status, smoking, BMI, T2DM or alcohol consumption.

\section{Caffeinated and decaffeinated coffee}

Four studies reported RRs of HCC specifically for decaffeinated coffee consumption. ${ }^{15} 212232$ No single study reported a statistically significant association between HCC and decaffeinated coffee consumption. Three cohort studies, ${ }^{1521} 22$ involving approximately 750000 participants and 800 cases, reported dose-response RRs or RRs for $>2$ consumption categories. The pooled RR of
HCC for two extra cups per day was 0.86 (95\% CI 0.74 to 1.00; three studies). Only two studies, involving approximately 850000 participants and 900 cases, reported RRs of HCC according to caffeinated coffee consumption in a manner suitable for dose-response analysis. ${ }^{1522}$ The pooled RR of HCC for an extra two cups of caffeinated coffee was 0.73 (95\% CI 0.63 to 0.85$)$.

\section{DISCUSSION}

In our meta-analysis of 18 cohort studies, involving 2272642 participants and 2905 cases, and 8 case-control studies, involving 1825 cases and 4652 controls, increasing coffee consumption by two cups per day was associated with a $35 \%$ reduction in the risk of HCC (RR $0.65 ; 95 \%$ CI 0.59 to 0.72 ). This is similar to previous meta-analyses. ${ }^{1325}$ In a subset of studies, the association was not significantly different in participants with pre-existing CLD at baseline, some of whom had cirrhosis. This is an important finding as the absolute risk of HCC in cirrhosis is high but may be more than halved by five cups per day of coffee compared with none (figure 3). The association was also not significantly different for the main exposures 


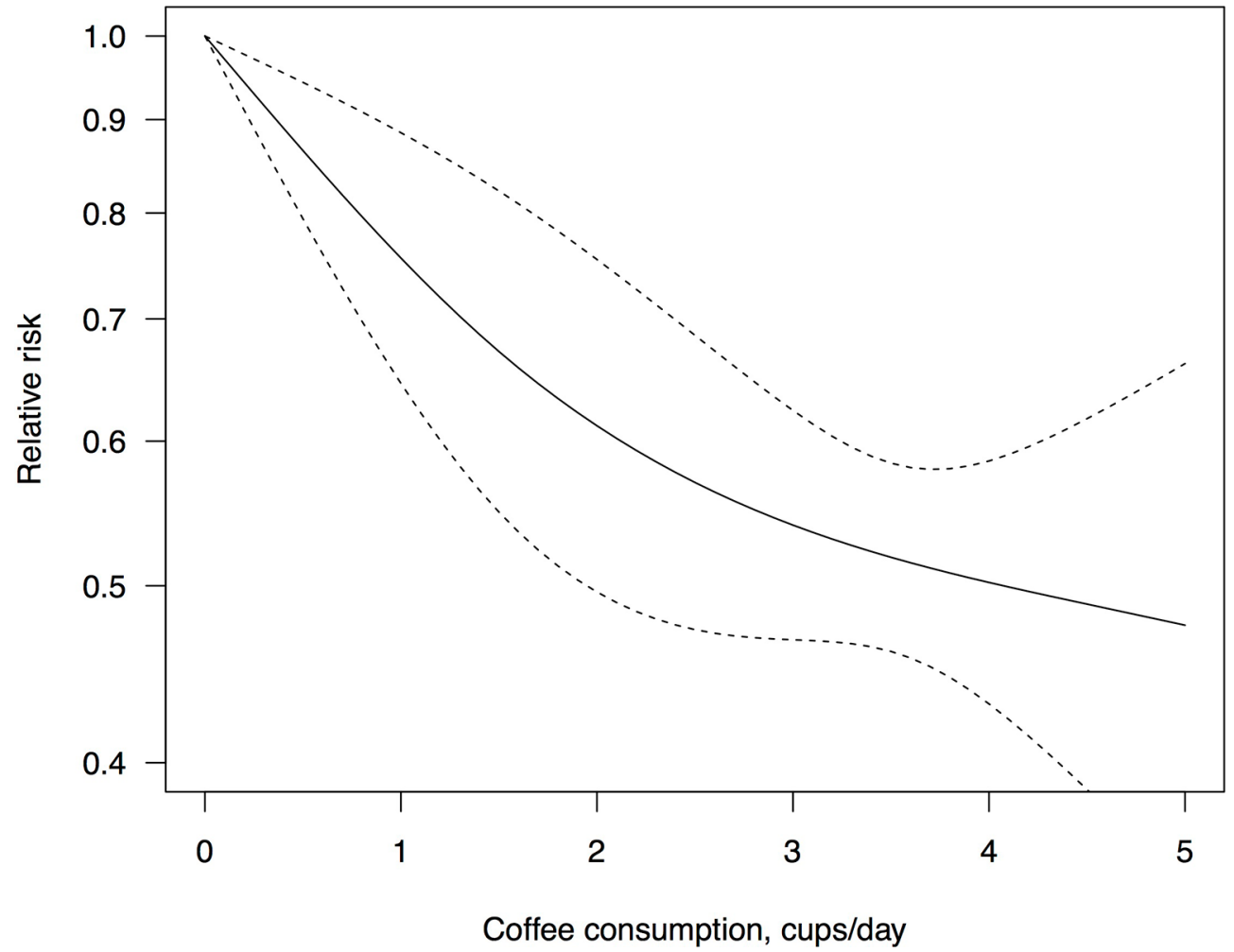

Figure 3 Results of a cubic spline dose-response meta-analysis of the association between coffee and hepatocellular carcinoma.

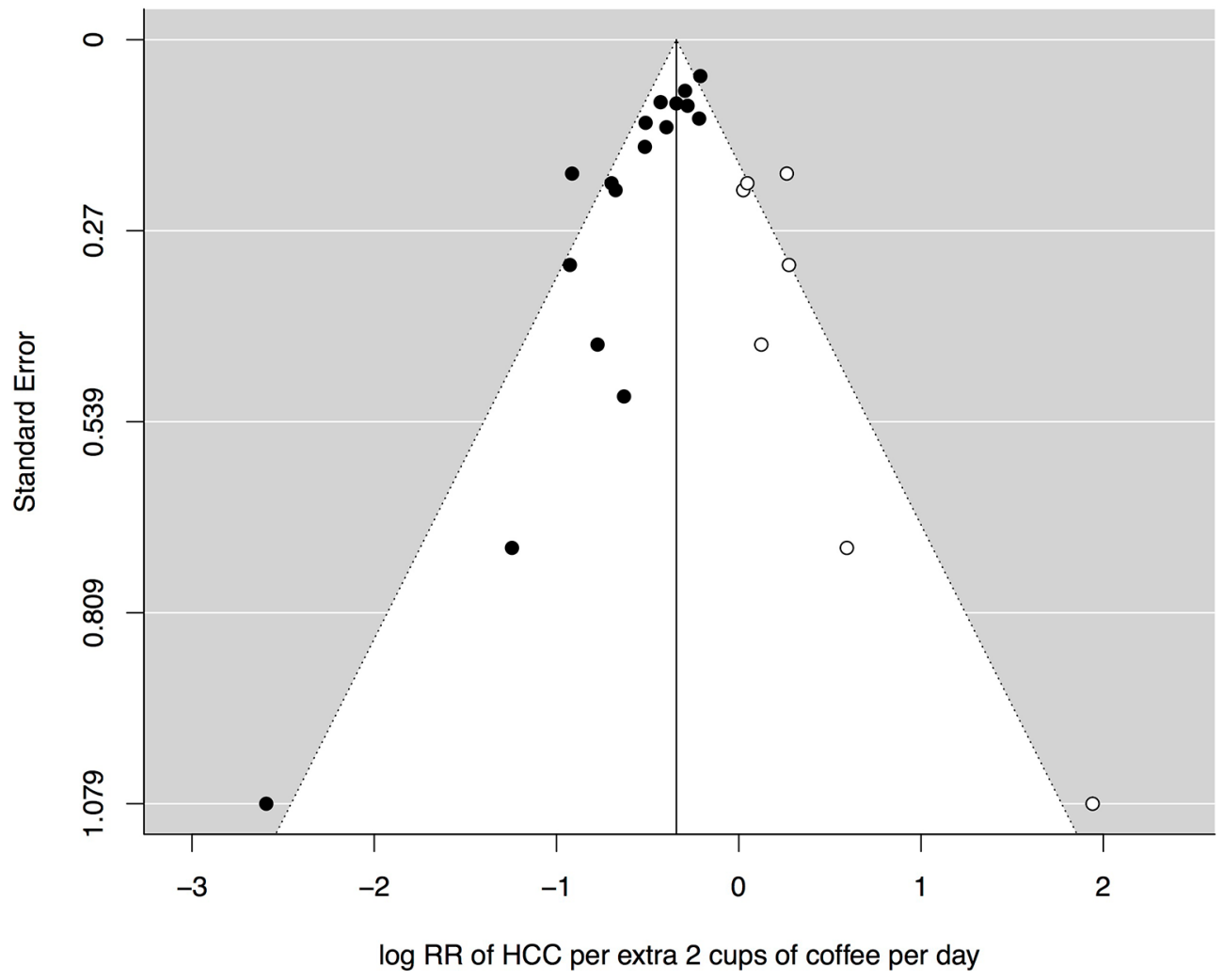

Figure 4 Filled funnel plot for the risk of HCC per extra two cups of coffee daily. Black circles represent the included studies found by our search, while white circles represent the 'missing' unpublished studies detected in the trim-and-fill analysis. HCC, hepatocellular carcinoma; RR, relative risk. 

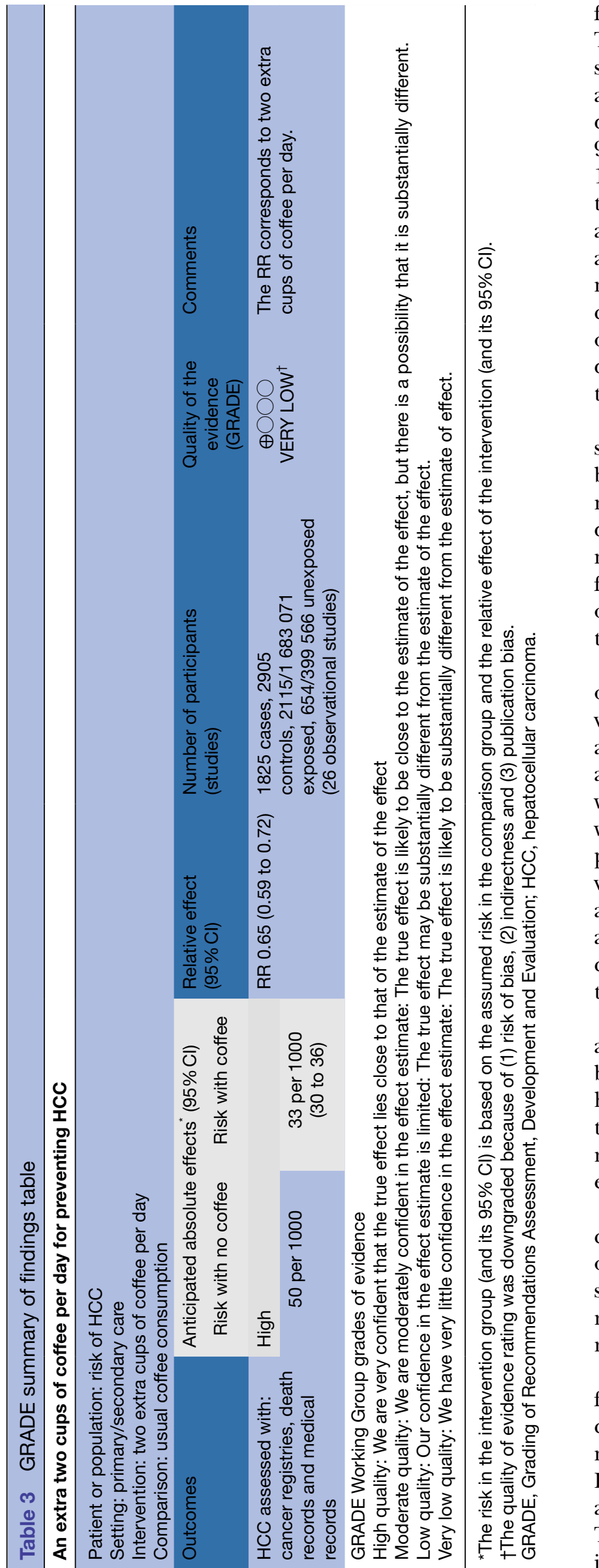

for HCC: high alcohol consumption, smoking, high BMI, T2DM and HBV/HCV. ${ }^{46}$ Data from the few studies that specified coffee type showed that increasing caffeinated and decaffeinated coffee consumption by two cups per day was associated with reductions of $27 \%$ (RR 0.73, $95 \%$ CI 0.63 to 0.85 ) and $14 \%$ (RR $0.86,95 \%$ CI 0.74 to $1.00)$ in the risk of HCC. This is the strongest evidence to date of an association between decaffeinated coffee and HCC. It may be important for developing coffee as a lifestyle intervention in CLD, as decaffeinated coffee might be more acceptable to those who do not drink coffee or who limit their coffee consumption because of caffeine-related symptoms. However, the benefits of decaffeinated coffee appear to be smaller and less certain than for caffeinated coffee.

Other major strengths of this meta-analysis are the systematic approach used to calculate a dose-response between coffee and HCC and the inclusion of a large number of participants and cases, representing a range of demographic groups (eg, gender, nationality, etc) and the main risk factors for HCC. We did not detect effect modification by baseline CLD and HCC aetiology, although our analysis was limited by the small number of studies that provided the necessary data for these analyses.

The main limitation is that all the included studies were observational, and thus we cannot infer causation. Observational studies are susceptible to bias and confounding, and case-control studies are at particular risk of selection and information bias. In the case-control studies, cases were mostly from hospital admissions or clinic records, which may not be representative of all HCC. Not all patients with HCC are admitted to hospitals, and individual factors associated with likelihood to attend clinic and/or to participate in a research study may be associated with coffee consumption or other risk factors (and confounders) for HCC. In addition, because of the need to interview participants, dead cases were not included.

The use of hospital controls in all except one study may also have introduced bias. First, there are associations between coffee drinking and a large number of other health conditions. ${ }^{47}$ Second, hospitals vary in the scale of their catchment areas and so hospital controls may not be representative of the populations from which cases arose, especially in areas where HCC care is highly specialised.

Among the cohorts, some studies used primary liver cancer as an outcome, whereas others used HCC. All but one cohort study used cancer registries to identify cases, sometimes in combination with death records. Cancer registries are more robust for ascertainment than death records.

Residual confounding likely existed in all studies from hidden factors and misclassification of measured confounders. However, adjustment for confounders had minimal effect on the association between coffee and HCC, suggesting residual effects will be small. All studies adjusted for alcohol, but several did not adjust for BMI, T2DM and HBV/HCV. Coffee was associated with alcohol in some studies, so failure to capture alcohol robustly 
might underestimate the inverse association between coffee and HCC. ${ }^{1541}$ The cohorts generally did not adjust for $\mathrm{HBV} / \mathrm{HCV}$ despite it being a major risk factor for HCC, but prevalence was likely low and we found no evidence of an effect of $\mathrm{HBV} / \mathrm{HCV}$ infection on the association between coffee and HCC.

The measurement of coffee consumption may also have introduced bias in case-control studies due to recall bias. Belief that coffee was harmful may have led to overestimation of consumption in cases. However, cases may have reduced coffee consumption because liver disease slows caffeine metabolism. ${ }^{48}$ One study used for baseline the consumption at 2 years before HCC diagnosis, ${ }^{32}$ when decades before may have been more appropriate. Another study ${ }^{43}$ reported RRs of HCC according to consumption preidentification and postidentification of liver disease; the weaker preidentification estimates were used in the meta-analysis, with minimal effect on the overall pooled RR.

In the cohorts, baseline CLD may have been present in cases given the short follow-up time of some cohorts compared with the long time for HCC to develop. However, we looked at a number of cohorts that presented data stratified by baseline CLD status and found no significant effect on the association between coffee and HCC. Setiawan et al found that the RR of HCC for two or more cups of coffee daily compared with none remained comparable in magnitude and statistically significant when deaths in the first 2 years were excluded. Lai et al found that the RR of HCC for an extra cup of coffee per day was $0.81(95 \%$ CI 0.66 to 0.98 ) in the first 10 years and 0.83 (95\% CI 0.71 to 0.96$)$ in the final 10 years of the study. Bamia et al, ${ }^{21}$ $\mathrm{Hu} e t a l^{8}$ and Shimazu et $a l^{39}$ reported similar findings. Thus, drinking coffee appeared to protect against HCC in participants with varying levels of undiagnosed CLD at baseline.

Our method of estimating median consumption in the reported consumption categories may have exaggerated the effect size. There was also a lack of data in most individual studies for higher levels of coffee consumption (eg, five cups per day or above). As a result, we had limited ability to detect an upper threshold beyond which increasing consumption no longer provides any benefit with regard to the risk of HCC. This is evident from figure 3, which shows rapidly widening CIs above four cups of coffee per day.

There was statistically significant heterogeneity between the studies; in a meta-regression analysis, it was not significantly associated with publication year, length of follow-up (cohorts only), percentage of alcohol abstainers, age or gender of participants.

Heterogeneity might be due to how consumption of coffee was measured. The included studies asked participants to estimate coffee consumption, usually by selecting from a list of predefined categories in food frequency questionnaires. Different categories may have influenced participants' responses. There may be variation in the size of cups, preparation (eg, boiled vs filtered) and caffeine content; 'coffee' was taken to be the pattern of use prevalent in the particular study population. Proportions of decaffeinated coffee drinkers varied markedly and were very low in certain countries (eg, Japan and Finland). ${ }^{33} 38$ Higher proportions of decaffeinated coffee drinkers, such as in the USA, ${ }^{22}$ may have attenuated the overall effect size given the weaker association found here between decaffeinated coffee and HCC.

Language bias cannot be excluded as we only included English studies, although studies found in the search were mostly in English. Generally, evidence of a significant influence in meta-analyses of language bias is weak. ${ }^{49}$ Studies published in non-English journals may also be less rigorous and report bigger effect estimates. ${ }^{50}$ Thus, our inclusion of English studies only is not likely to have introduced significant bias. Finally, we found evidence of publication bias using Egger's test. Adjusting for smaller unpublished studies pushed the effect size towards null, but it remained statistically significant.

Our study adds to the weight of evidence considered by the IARC and WCRF that coffee is protective against HCC. However, when assessed under the GRADE criteria, the quality of evidence supporting coffee for the prevention of HCC was still 'very low'. This was mainly because of the lack of randomised trials, evidence of publication bias, and the fact that 'coffee', which has various formulations with different chemical properties, is not well defined.

\section{Mechanism of action}

As discussed in detail in previous work, ${ }^{1851}$ there is biological plausibility of a protective effect of coffee against HCC. The fact that we found no significant effect of aetiology albeit in a subset of studies suggests that the apparent protective mechanism acts via a common pathway, such as the development of cirrhosis. Eighty to ninety per cent of cases of HCC develop on a background of cirrhosis, ${ }^{51}$ and several studies and a meta-analysis have reported an inverse association between coffee and cirrhosis. ${ }^{18}$ Coffee may possess direct anticarcinogenic properties, which is supported by our finding that the association of coffee and HCC was seen in those with pre-existing CLD, including cirrhosis. Our findings suggest a central role for caffeine, given that the association was weaker for decaffeinated coffee. Caffeine reduces HCC cell proliferation. ${ }^{52}$ Cafestol and kahweol increase activity of phase 2 liver enzymes, which may improve metabolism and excretion of carcinogens, ${ }^{53}$ and compounds including polyphenols may ameliorate oxidative DNA damage. However, cafestol and kahweol are present only in minimal quantities in instant and filtered coffee,$^{55}$ and these varieties are popular in Japan and Finland, respectively, where studies included in this meta-analysis show inverse associations with HCC. ${ }^{33} 38$ Other specific mechanisms of protection might include inhibition of hepatitis virus activity ${ }^{56}$ and prevention of T2DM. $^{38}$

Coffee purportedly possesses a range of health effects in addition to those on the liver, including lower incidences 
of neurological diseases, various cancers and any-cause mortality. ${ }^{47}$ However, randomised trials are needed of interventions to support patients at risk of HCC to increase coffee consumption before recommending an increase given the examples in other areas of where RCTs have shown observational data to be incorrect and the global scale and ubiquity of coffee consumption. ${ }^{57}$ The potential harms of coffee also require further investigation, including the reported increased risk of lung cancer and bone fractures ${ }^{47}$ and the deleterious effect on cholesterol, which could potentially exacerbate the already increased risk of CVD associated with certain types of liver disease. ${ }^{58}$

In summary, this study has shown that an extra two cups of coffee per day is associated with a one-third reduction in the RR of HCC. Our findings are significant given the increasing incidence of HCC and the overall poor prognosis of this condition. Randomised trials should investigate the effectiveness of increasing coffee consumption in those at risk of HCC including patients with existing CLD.

Contributors The study was conceived by all authors; The search was performed by $\mathrm{OK}$. The studies were reviewed and selected by RB and $\mathrm{OK}$. The quality of evidence assessment was performed by $0 \mathrm{~K}$. The risk of bias assessment was performed by JP and OK. The data were extracted and checked by OK and RB, respectively. The statistical analysis was performed by OK. The manuscript was drafted by $\mathrm{OK}$ and reviewed and amended by all authors. JP is guarantor.

Funding This research received no specific grant from any funding agency in the public, commercial or not-for-profit sectors.

Provenance and peer review Not commissioned; externally peer reviewed.

Data sharing statement № additional data are available.

Open Access This is an Open Access article distributed in accordance with the Creative Commons Attribution Non Commercial (CC BY-NC 4.0) license, which permits others to distribute, remix, adapt, build upon this work non-commercially, and license their derivative works on different terms, provided the original work is properly cited and the use is non-commercial. See: http://creativecommons.org/ licenses/by-nc/4.0/

(C) Article author(s) (or their employer(s) unless otherwise stated in the text of the article) 2017. All rights reserved. No commercial use is permitted unless otherwise expressly granted.

\section{REFERENCES}

1. World Cancer Research Fund International. Liver cancer statistics. http://www.wcrf.org/int/cancer-facts-figures/data-specific-cancers/ liver-cancer-statistics (accessed 3rd Jan 2016)

2. International Agency for Research on Cancer. Liver Cancer. Estimated incidence, mortality and prevalence worldwide in 2012. http://globocan.iarc.fr/Pages/fact_sheets_cancer.aspx (accessed 3rd Jan 2016).

3. El-Serag HB, Rudolph KL. Hepatocellular carcinoma: epidemiology and molecular carcinogenesis. Gastroenterology 2007;132:2557-76.

4. Kawada N, Imanaka K, Kawaguchi T, et al. Hepatocellular carcinoma arising from non-cirrhotic nonalcoholic steatohepatitis. $J$ Gastroenterol 2009;44:1190-4.

5. International Agency for Research on Cancer. Cancer incidence, mortality and prevalence worldwide. http://tinyurl.com/heoxe2u (accessed 3rd Jan 2016).

6. Pang TC, Lam VW. Surgical management of hepatocellular carcinoma. World J Hepatol 2015;7:245-52.

7. Jemal A, Bray F, Center MM, et al. Global cancer statistics. $C A$ Cancer J Clin 2011;61:69-90.

8. Ponte S. The 'Latte Revolution'? regulation, markets and consumption in the Global Coffee Chain. World Dev 2002;30:1099-122.

9. Ludwig IA, Clifford MN, Lean ME, et al. Coffee: biochemistry and potential impact on health. Food Funct 2014;5:1695-717.
10. Saab S, Mallam D, Cox GA, et al. Impact of coffee on liver diseases: a systematic review. Liver Int 2014;34:495-504.

11. World Cancer Research Fund International/American Institute for Cancer Research. Continuous Update Project Report: diet, Nutrition, Physical Activity and liver Cancer. 2015 wcrf.org/sites/default/files/ Liver-Cancer-2015-Report.pdf.

12. Loomis D, Guyton KZ, Grosse Y, et al. Carcinogenicity of drinking coffee, mate, and very hot beverages. Lancet Oncol 2016;17:877-8.

13. Bravi $F$, Tavani $A$, Bosetti $C$, et al. Coffee and the risk of hepatocellular carcinoma and chronic liver disease: a systematic review and meta-analysis of prospective studies. Eur $J$ Cancer Prev 2016.

14. Arauz J, Moreno MG, Cortés-Reynosa P, et al. Coffee attenuates fibrosis by decreasing the expression of TGF- $\beta$ and CTGF in a murine model of liver damage. J App/ Toxicol 2013;33:970-9.

15. Setiawan VW, Wilkens LR, Lu SC, et al. Association of coffee intake with reduced incidence of liver cancer and death from chronic liver disease in the US multiethnic cohort. Gastroenterology 2015;148:118-25.

16. Xiao Q, Sinha R, Graubard Bl, et al. Inverse associations of total and decaffeinated coffee with liver enzyme levels in National Health and Nutrition Examination Survey 1999-2010. Hepatology 2014;60:2091-8.

17. Ding M, Bhupathiraju SN, Chen M, et al. Caffeinated and decaffeinated coffee consumption and risk of type 2 diabetes: a systematic review and a dose-response meta-analysis. Diabetes Care 2014;37:569-86.

18. Kennedy OJ, Roderick P, Buchanan R, et al. Systematic review with meta-analysis: coffee consumption and the risk of cirrhosis. Aliment Pharmacol Ther 2016;43:562-74.

19. Ottawa Hospital Research Institute. The Newcastel-Ottawa Scale (NOS) for assessing the quality of nonrandomised studies in metaanalyses. www.ohri.ca/programs/clinical_epidemiology/oxford.asp (accessed 14th December 2015).

20. Schünemann H, Brożek J, Oxman G. Handbook for grading the quality of evidence and the strength of recommendations using the GRADE approach. 2013. www.guidelinedevelopment.org/handbook/ (accessed 4th Aug 2015).

21. Bamia C, Lagiou P, Jenab M, et al. Coffee, tea and decaffeinated coffee in relation to hepatocellular carcinoma in a European population: multicentre, prospective cohort study. Int $J$ Cancer 2015;136:1899-908.

22. Petrick JL, Freedman ND, Graubard BI, et al. Coffee Consumption and risk of hepatocellular carcinoma and intrahepatic cholangiocarcinoma by sex: the liver Cancer pooling project. Cancer Epidemiol Biomarkers Prev 2015;24:1398-406.

23. Lai GY, Weinstein SJ, Albanes D, et al. The association of coffee intake with liver cancer incidence and chronic liver disease mortality in male smokers. Br J Cancer 2013;109:1344-51.

24. Greenland S, Longnecker MP. Methods for trend estimation from summarized dose-response data, with applications to meta-analysis. Am J Epidemiol 1992;135:1301-9.

25. Larsson SC, Wolk A. Coffee consumption and risk of liver cancer: a meta-analysis. Gastroenterology 2007;132:1740-5.

26. Orsini N, Li R, Wolk A, et al. Meta-analysis for linear and nonlinear dose-response relations: examples, an evaluation of approximations, and software. Am J Epidemiol 2012;175:66-73.

27. Deeks JJ, Higgins JPT, Altman DG, Chapter 9: Analysing data and undertaking meta-analyses. In: Higgins JPT, Green S, eds. Cochrane Handbook for Systematic Reviews of Interventions Version 5.1.0 (updated March 2011). www.handbook.cochrane.org.

28. Greenland S. Quantitative methods in the review of epidemiologic literature. Epidemiol Rev 1987;9:1-30.

29. Jonathan ESA, Egger M, Moher D, et al; Addressing reporting biases. In: Higgins J, Green S, eds. Cochrane handbook for systematic reviews of interventions. Cochrane Collaboration, 2008:1-33.

30. Viechtbauer W. Conducting Meta-Analyses in $R$ with the metafor package. J Stat Softw 2010;36:1-48

31. Crippa A, Orsini N. Multivariate Dose-Response Meta-Analysis: the dosresmeta $R$ package. J Stat Softw 2016;72:1-15.

32. Montella M, Polesel J, La Vecchia C, et al. Coffee and tea consumption and risk of hepatocellular carcinoma in Italy. Int $J$ Cancer 2007:120:1555-9.

33. Tanaka K, Hara M, Sakamoto T, et al. Inverse association between coffee drinking and the risk of hepatocellular carcinoma: a casecontrol study in Japan. Cancer Sci 2007:98:214-8.

34. Wakai K, Kurozawa Y, Shibata A, et al. Liver cancer risk, coffee, and hepatitis $C$ virus infection: a nested case-control study in Japan. $B r J$ Cancer 2007;97:426-8. 
35. Kurozawa $\mathrm{Y}$, Ogimoto I, Shibata A, et al. Coffee and risk of death from hepatocellular carcinoma in a large cohort study in Japan. $\mathrm{Br} J$ Cancer 2005;93:607-10.

36. Inoue M, Yoshimi I, Sobue T, et al. Influence of coffee drinking on subsequent risk of hepatocellular carcinoma: a prospective study in Japan. J Natl Cancer Inst 2005;97:293-300.

37. Gallus S, Bertuzzi M, Tavani A, et al. Does coffee protect against hepatocellular carcinoma? Br J Cancer 2002;87:956-9.

38. Hu G, Tuomilehto J, Pukkala E, et al. Joint effects of coffee consumption and serum gamma-glutamyltransferase on the risk of liver cancer. Hepatology 2008;48:129-36.

39. Shimazu T, Tsubono Y, Kuriyama S, et al. Coffee consumption and the risk of primary liver cancer: pooled analysis of two prospective studies in Japan. Int J Cancer 2005;116:150-4.

40. Zamora-Ros R, Luján-Barroso L, Bueno-de-Mesquita HB, et al. Tea and coffee consumption and risk of esophageal cancer: the European prospective investigation into cancer and nutrition study. Int J Cancer 2014;135:1470-9.

41. Johnson S, Koh WP, Wang R, et al. Coffee consumption and reduced risk of hepatocellular carcinoma: findings from the Singapore Chinese Health Study. Cancer Causes Control 2011;22:503-10.

42. Gelatti U, Covolo L, Franceschini M, et al. Coffee consumption reduces the risk of hepatocellular carcinoma independently of its aetiology: a case-control study. J Hepatol 2005;42:528-34.

43. Ohfuji S, Fukushima W, Tanaka T, et al. Coffee consumption and reduced risk of hepatocellular carcinoma among patients with chronic type C liver disease: a case-control study. Hepatol Res 2006;36:201-8.

44. Stucker I, N'Kontchou G, Loriot MA, et al. Does coffee drinking protect cirrhotic patients against hepatocellular carcinoma? Hepatology 2006:44:501A-01A.

45. Leung WW, Ho SC, Chan HL, et al. Moderate coffee consumption reduces the risk of hepatocellular carcinoma in hepatitis $\mathrm{B}$ chronic carriers: a case-control study. J Epidemiol Community Health 2011;65:556-8.

46. Lee MH, Yang HI, Lu SN, S-n L, et al. Hepatitis C virus genotype $1 \mathrm{~b}$ increases cumulative lifetime risk of hepatocellular carcinoma. Int $J$ Cancer 2014;135:1119-26.
47. Cano-Marquina A, Tarín JJ, Cano A. The impact of coffee on health. Maturitas 2013;75:7-21.

48. Rodopoulos N, Wisén O, Norman A. Caffeine metabolism in patients with chronic liver disease. Scand J Clin Lab Invest 1995;55:229-42.

49. Morrison A, Polisena J, Husereau D, et al. The effect of Englishlanguage restriction on systematic review-based meta-analyses: a systematic review of empirical studies. Int J Technol Assess Health Care 2012;28:138-44.

50. Jüni $P$, Holenstein $F$, Sterne $J$, et al. Direction and impact of language Bias in meta-analyses of controlled trials: empirical study. Int $J$ Epidemiol 2002;31:115-23.

51. Fattovich G, Stroffolini T, Zagni I, et al. Hepatocellular carcinoma in cirrhosis: incidence and risk factors. Gastroenterology 2004;127:S35-50.

52. Okano J, Nagahara T, Matsumoto K, et al. Caffeine inhibits the proliferation of liver cancer cells and activates the MEK/ERK/EGFR signalling pathway. Basic Clin Pharmacol Toxicol 2008;102:543-51.

53. Cavin C, Holzhaeuser D, Scharf G, et al. Cafestol and kahweol, two coffee specific diterpenes with anticarcinogenic activity. Food Chem Toxicol 2002;40:1155-63.

54. Huber WW, Scharf G, Rossmanith W, et al. The coffee components kahweol and cafestol induce gamma-glutamylcysteine synthetase, the rate limiting enzyme of chemoprotective glutathione synthesis, in several organs of the rat. Arch Toxicol 2002;75:685-94.

55. Urgert R, van der Weg G, Kosmeijer-Schuil TG, et al. Levels of the Cholesterol-Elevating Diterpenes Cafestol and Kahweol in Various Coffee Brews. J Agric Food Chem 1995;43:2167-72.

56. Batista MN, Carneiro BM, Braga AC, et al. Caffeine inhibits hepatitis C virus replication in vitro. Arch Virol 2015;160:399-407.

57. Baron JA, Barry EL, Mott LA, et al. A trial of calcium and vitamin $\mathrm{D}$ for the Prevention of colorectal adenomas. N Engl J Med 2015;373:1519-30.

58. Targher G, Day CP, Bonora E. Risk of cardiovascular disease in patients with nonalcoholic fatty liver disease. N Engl J Med 2010;363:1341-50. 